this synthesis, it was decided to repeat this work. The acids described as $(A),(B)$ and $(C)$ were isolated in accordance with this author's directions ${ }^{1}$, but no trace of the fourth isomer could be obtained. The acids $(A)$ and $(C)$ proved identical with the two acids obtained by Higson and Thorpe's method, but the third isomer $(B)$ was actually a mixture of $(A)$ and $(C)$. We thus arrived independently at the conclusion of Goldschmidt and Grafinger, that there are only two stereoisomeric forms of 1-carboxy-4-methylcyclohexane-1-acetic acid.

It may be added that we have been unable to isolate the carboxy-3:3-dimethylcyclohexane-1-acetic acid obtained from 3:3-dimethylcyclohexanone, in more than one form.

\section{R. D. Desar. \\ R. F. Hunter.}

Department of Chemistry, Muslim University, Aligarh.

Sept. 20.

${ }^{1}$ J. Indian Chem. Soc., 8, 277; 1931.

\section{Spectrum Analysis}

THE term 'spectrum analysis' was originally used to denote the analysis of substances by means of their spectra, and it would probably still be interpreted in this sense by most experimental spectroscopists. To those not accustomed to this usage, how ever, it seems to connote the analysis of the structure of a spectrum, for example, its resolution into series or multiplets.

Might it not be well to abandon the term 'spectrum analysis' and substitute one free from this ambiguity?

Prof. E. N. da C. Andrade suggests 'spectrochemical analysis' and Prof. W. E. Curtis 'spectroscopic analysis'. For the time being, those associated with me here will endeavour to use the former phrase, but I should be glad, as doubtless would others, if through the medium of your pages other opinions could be brought to light for our guidance.

$$
\text { F. Twyman. }
$$

Adam Hilger, Ltd.,
98 Kings Road,
Camden Road,
London, N.W.1.
Sept. 19.

Adam Hilger, Ltd.,

98 Kings Road, Sept. 19.

\title{
Points from Foregoing Letters
}

THE reviewer of "The Diary of Robert Hooke" is criticised by Mr. T. E. James for his description of Henry Oldenburg as an "oblique, intriguing and toadying individual". Mr. James ascribes the absence of Hooke's portrait in the Royal Society to Hooke's "fickle physical condition" probably making him a difficult 'sitter'. Dr. R. T. Gunther complains of the statement that the biographer, Richard Waller, "apparently had little personal knowledge of Hooke", and points to the recently published later diaries, which indicate that Waller was frequently in the company of Hooke. The reviewer, Prof. E. N. da C. Andrade, gives some of the historical evidence upon which he has based his unfavourable opinion of Oldenburg and asks for counter-evidence. He directs attention to diary entries which suggest that a painting of Hooke by "Bonust" may perhaps be in existence.

Dr. Dingle has distinguished three aspects of probability : (i) the ordinary everyday aspect or 'likelihood' (such as the odds in betting on a horse race), usually the result of instinctive evaluation rather than of calculation; (ii) the physicist's probability, measured from past events (the favourite wins once in ten times); (iii) the mathematically derived 'chance' of future occurrences derived by combining data from independent sources such as (i) and (ii). Dr. Dingle has called the probability calculated in this way a symbolical function, meaningless from the operational point of view. W. Barrett. and T. Smith take exception to the reasoning in one example adduced by Dr. Dingle to illustrate his view. Dr. Dingle restates his case in general terms and points out that the objections raised are due to misunderstandings.

Graphs indicating first a rise and then a decrease in the attenuation of radio waves in two definite directions up to a distance of $4,800 \mathrm{~m}$. are submitted by Dr. S. R. Khastgir, D. N. Chandhuri and B. Sen Gupta. The authors point out that the experimental results can be accurately accommodated by
Sommerfeld's formula, making use of Rolf's graphs, by giving to the two main constants values agreeing with laboratory determinations of the dielectric constants and electrical conductivities of soils of varying moisture content.

From spectroscopic and quantum-mechanical considerations, H. Lessheim and Prof. R. Samuel calculate the heat of dissociation of carbon monoxide (by Kaplan's method) and find a somewhat lower value, 10.45 v.e., than that recently calculated by Goldfinger, Lasareff and Rosen in a different manner. The corresponding heat of sublimation of carbon comes to $155 \cdot 7 \mathrm{k} . c a l . / \mathrm{mol}$. at $25^{\circ} \mathrm{C}$.

The presence of oxygen in the sun's chromosphere up to a height of $3 \cdot 5^{\prime \prime}$ or more has been observed by T. Royds by means of a spectrograph. The infra-red oxygen spectrum lines, $7771,7774,7775$, are clearly recorded.

Referring to a recent leading article in NATURE, in which the various factors needed for the development of a social science were discussed, Alan Blair points out that Acts of Parliament are equivalent to social experiments; although perfect 'controls' to such experiments are lacking, one may compare the effects produced with conditions before the passage of such Acts and with the effect of similar laws elsewhere.

Prof. Cannon, by staining with anilin blue a specimen of the fairy-shrimp, Chirocephalus, has observed an amorphous mass on either side of the upper border covering the mouth (labrum) over the ventral food groove, and has concluded that it was secreted by the labral glands. Miss K. W. Nicholson and Prof. C. M. Yonge, who believe that the labral glands have nothing to do with feeding but are effective in the formation of the cuticle, report an experiment in which a Chirocephalus was fed a suspension of carmine. After fixation, an amorphous mass similar to that observed by Cannon but due merely to the regurgitated contents of the gut was noted. 